\title{
Smart Sensor-based Interface Controlin the Mobile CloudEnvironment
}

\author{
Sanghyun Park ${ }^{1}$, Ilmin Kim ${ }^{2}$ and Jinsul kim ${ }^{3 *}$ \\ ${ }^{1,3}$ School of Electronics and Computer Engineering, Chonnam National University, \\ 77 Yongbong-ro, Buk-gu, Gwangju 500-757, Republic of Korea \\ ${ }^{2}$ Dept. of Computer Science, Hansung University, Samseongyoro-16 gil, \\ Seongbuk-gu, Seoul 136-792 \\ Sanghyun079@gmail.com ${ }^{1}$,ikim@hansung.ac.kr², jsworld@jnu.ac.kr *
}

\begin{abstract}
In this paper we propose the use of cloud services to delivery next-generation dynamic mobile interface. The improvement is focused on existing soft keyboard application. Through a simple sign-in process, the customized keyboard interfaced defined by user own can by synced, stored on the cloud and ready to be apply on other compatible devices of the same user in anytime. With cloud based interface, through specific username and password, various smart mobile devices are able to receive their custom interface. Also, we use Gyro Sensor and Wi-Fi direct to help the users freely share their layout through the internet.
\end{abstract}

Keywords: Hadoop, Customized Mobile Interface, Cloud Server, Gyro Sensor

\section{Introduction}

With the development of the Internet environment, users can connect to the Internet anytime, anywhere. In addition, due to the smart mobile devices, people are able to easily access information by using the Internet. According to the IT research firm eMarketer, there are approximately 45 billion smart mobile phone users all over the world in 2014 [1]. A smart mobile phone is no longer simple device for calling but support a variety of tasks and functions [2]. Smart mobile phone has many features and used to suit their own tastes. The initial smart mobile phones products provided for changing the theme, the background or soft keyboard layout. Smart Mobile Phones OS's version is being updated, and supply increases, thus taking advantage of various applications and users can easily change the soft keyboard skin layout, icon, and background. By using previous applications, users can easily change the soft keyboard layouts, the app icon, and the background but only use the themes provided by the developer. As a result, these themes are usually used by ordinary people comfortably, but elder person and disabled person are uncomfortable to use them. This paper provides customized interfaces for a variety of users by using Web-based authoring tools, the interfaces can be created and offered easily. With only using a web browser and a mouse drag and click function which can produce interfaces may be shared with others. Also, we share the interface between users in an environment that does not have internet using Wi-Fi Direct and Gyro sensor, sharing the interface data.

\section{Related Works}

We have some upfront research to advance research and investigation. Figure 1 presentsan IP based virtual smartphone released in research paper in 2010 [3]. Theexisting Smartphones 
to use applications must go through the installation process. However, Figure 1 shows that without application installation, virtual environments for a variety of applications can be used. You can use the smart device's viewer application to use the application provided by the server. Because of the use of the applications in a virtual environment, there are advantages of security metrics, and the deleted application is not used or need to be upgraded. Also because the application does not operate internally, high-end applications can be used on high performance non-smartphones.

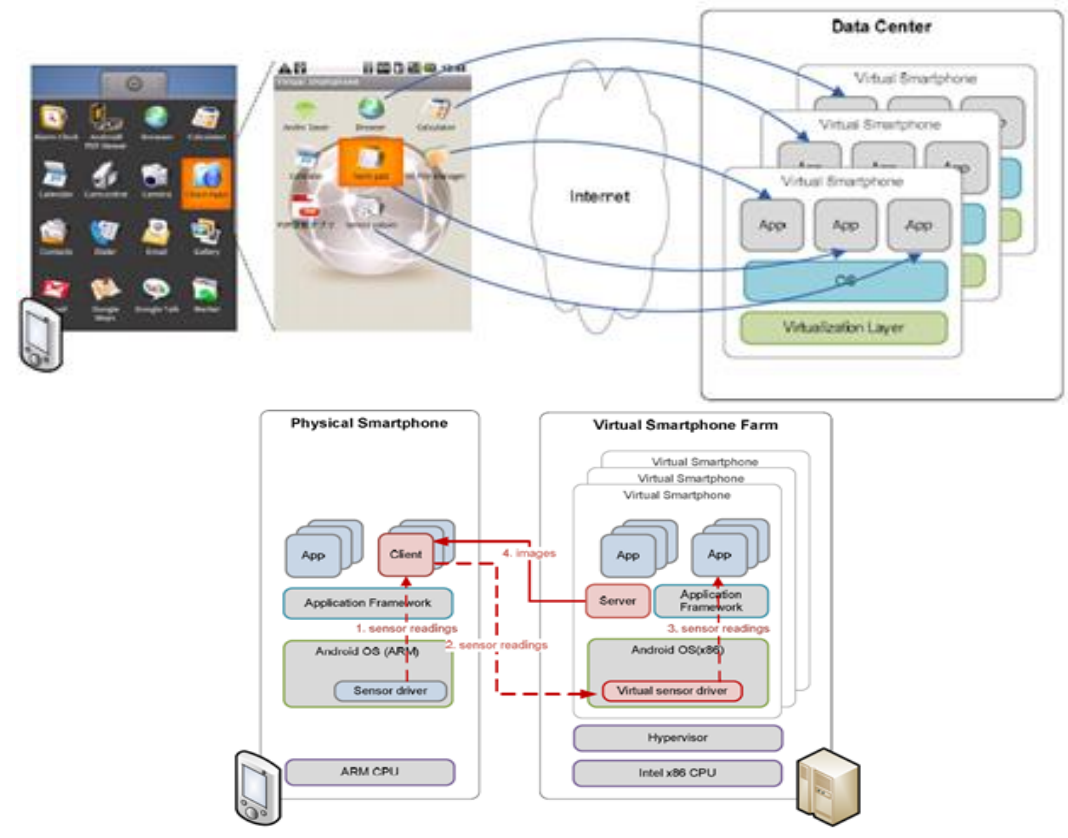

Figure 1. IP based Virtual Smartphone

Figure 2 shows a measure of the speed of FPS operation that occurs when the application graph. Android (x86) in a virtual environment to provide a workstation applications to speak, and Android (ARM) is a smart phone speaker. The paper does not work virtual applications on the user's smart phone FPS is the measure is not high, and does not use a lot of internal resources, the battery can be used for long. However, because the use of an application in real-time in a virtual environment to be supported smoothly wireless communication environment, has a disadvantage, if the wireless communication signal is low, and that the user's smartphone battery consuming. In addition, when using content such as images and words, picture, etc., a lot of FPSis not required, but motion images and video from the behavior quick FPS needed in order to receive a seamless service, we need to have wireless communication environment. When using a fast wireless communication continues to be very faster battery consumption. We propose in the paper a virtualization system interface to apply real-time communication in order to facilitate speed should be guaranteed, and data entered by the user must be the exact transmission conditions. When ensuring fast the communication environment, the conditions are met. However, if it is not a fast communication environment, errors cause a lot of concern. 


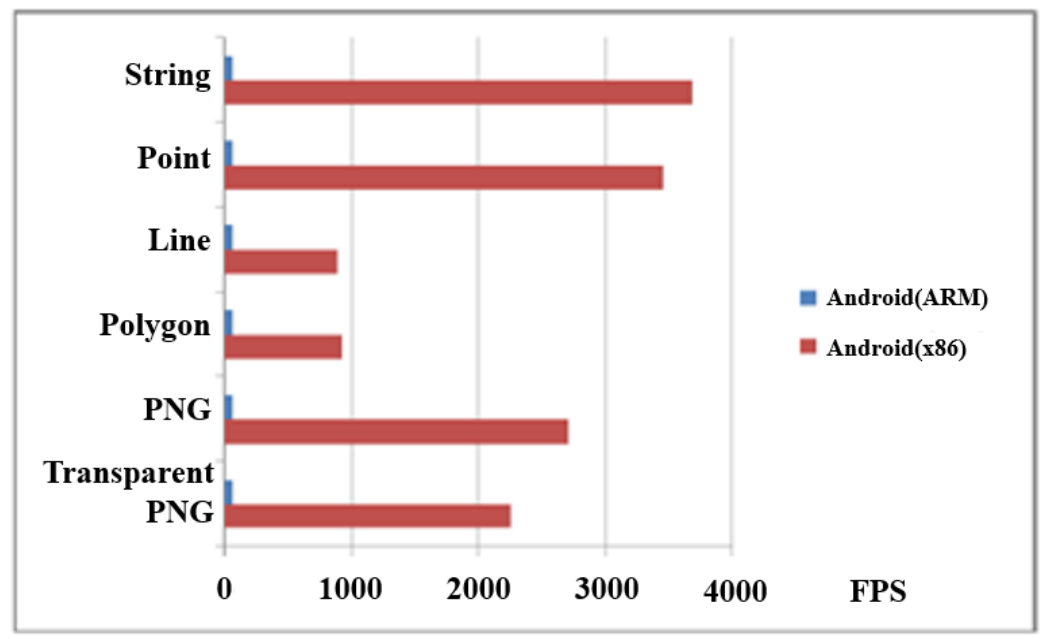

Figure 2. IP based Virtual Smartphone

Figure 3 shows the application developed in the United Kingdom for the visually impaired people tohelp send text messages easily [4]. This application is different from the General interface of the mobile devices that are composed of the Braille system. As shown in the figure, youuse six fingers for typing letters, and no need for much movement. In addition, with the application for the visually impaired,you can use your fingers to enter the Braille alphabet with voice output. Some experts say that practically the visually impaired peopleare slow, and it's hard for them to enter characters quickly. This application is a special application for the blind and cannot be used with a variety of people.

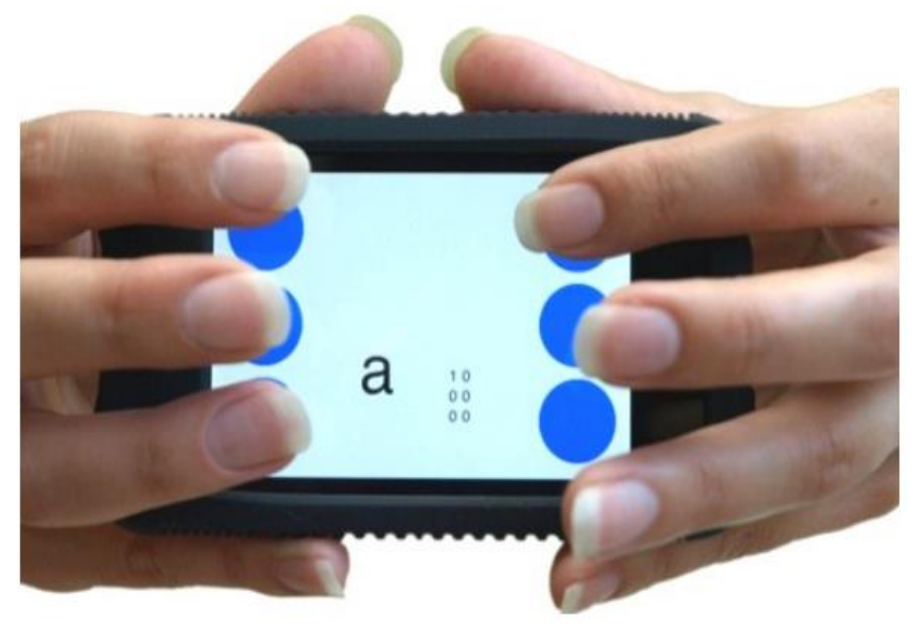

Figure 3. Braille Touch of Georgia Tech

Figure 4 shows the developedKii Keyboard application consisting of Swiftkey, Swype, Go keyboard, with a variety of functions in one package. This application allows users to make and use their desired interface, provides a framework user desired label, and after color setting, its' saved and applied. This application allows the user to create the desired interface, but the interface key positions do not change to a variety of positions and does not provide them with a variety of layout skins. Also, themadeinterface cannot shared with other users. 


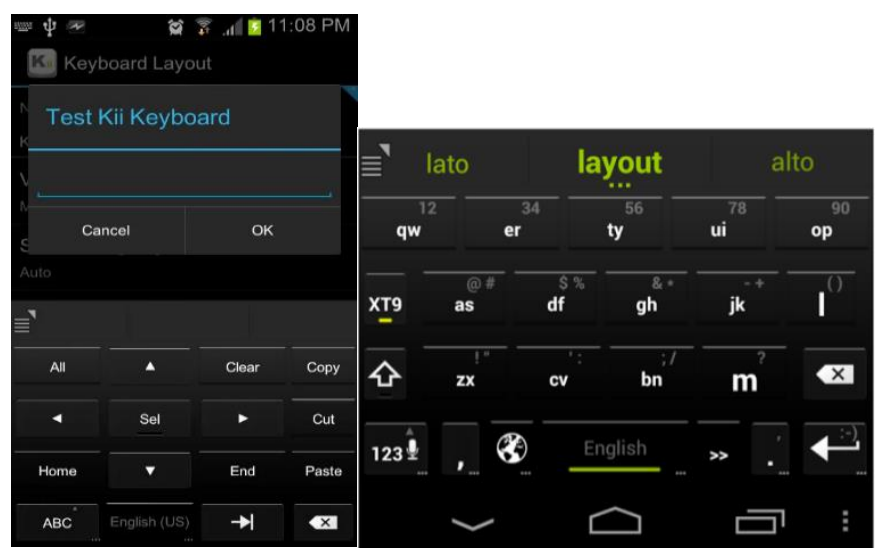

Figure 4. Kii Keyboard Application

In relation to smart mobile interface, there has been a variety of research and applications have been developed, but the optimized interfaces are not provided for a variety of people. We can use a cloud environment to provide an optimized interface for a wide variety of people. In addition, using the Gyro Sensor and Wi-Fi Direct, various interfaces can be made and shared in environments without internet.

\section{Mobile Interface Provider Structure}

The current smart mobile devices are supported from inside a keyboard interface, or user can change the skin layout using your app. Therefore, this paper uses a Web-based authoring Toolkit to provide an interface that users can easily create. The user through the cloud server can create and share interfaces by login to $3 \mathrm{G}$, LTE Wi-Fi environment, at anytime, anywhere. If there are 3 servers in a cloud environment, user data management server manage the user's data in the user's ID, password, and the information of the user according to the interface are used as identifiers for. User ID, using the same interface in a variety of smart mobile devices can be applied. Interface Management Server provides a variety of interfaces to manage their information, and each key value, key color, key size, location, and label value information. The interface management server not only stores information but also used for easy creation of user interface and administrator interface and the management server provides a web-based interface toolkit interface. The mapping server helps to make a quick search of the selected user interfaces, the user makes a request, and it checks if the user interface based on the information provided can be made. Retrieving data from the interface information takes a lot of time, but because of the usage of the mapping management server you can manage the entire data in the form of links, and get the desired data quickly. 


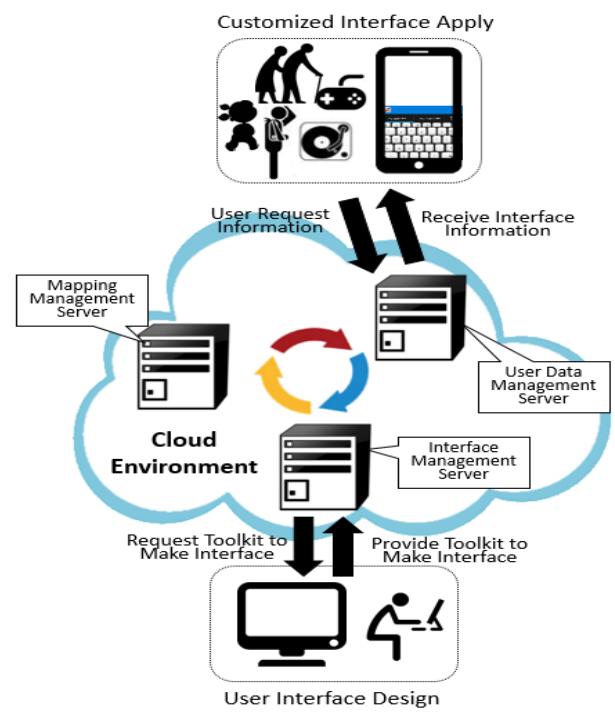

Figure 5. System Architecture of the Customized UI Provider

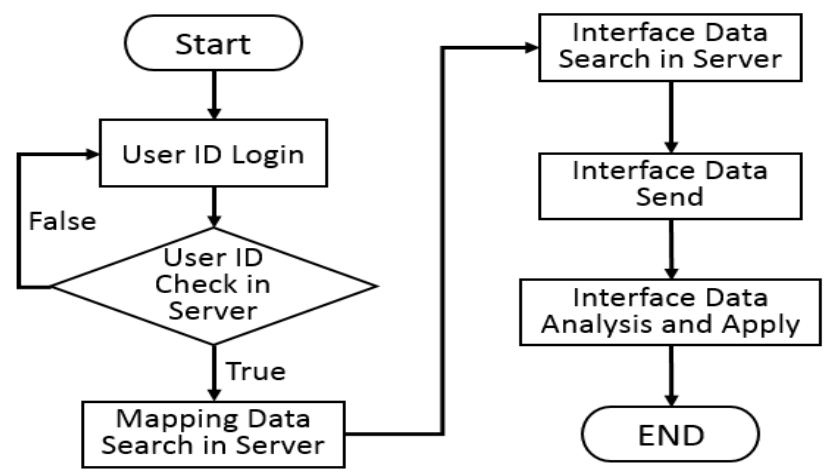

Figure 6. Customized Interface Provide Flowchart

Figure 6 shows a customized interface that receives the request. For users to receive a customized interface for smart mobile devices, they have to log on to the server. The user's ID and password is sent to the server, the server will check if the user is correct. If not, return to login, if the user is verified, using the mapping server the requested interface is searched from the server. Mapping data is not the actual data, rather than the quick search links to the data, search for links based on data retrieved from the server of the actual interface data. The data is sent in XML format file [5, 6], the user's smart mobile device retrieves the sent interface data and after analysis it is applied. Users can also store data request or store data in a manner similar to above. Users can use a Web-based Toolkit interface to create and save the interface and even if the file is not applied on the mapping server, the interface management server stores data of the interface. The mapping management serverapplies only user created interface and other users selected shared interfaces. The mapping management server does not have the entire data of the user created interface but the information of the applied interfaces on the smart mobile device. As show in Figure 6, the interface customization could be done through web interface and the customized interfaces could be shared among users. 


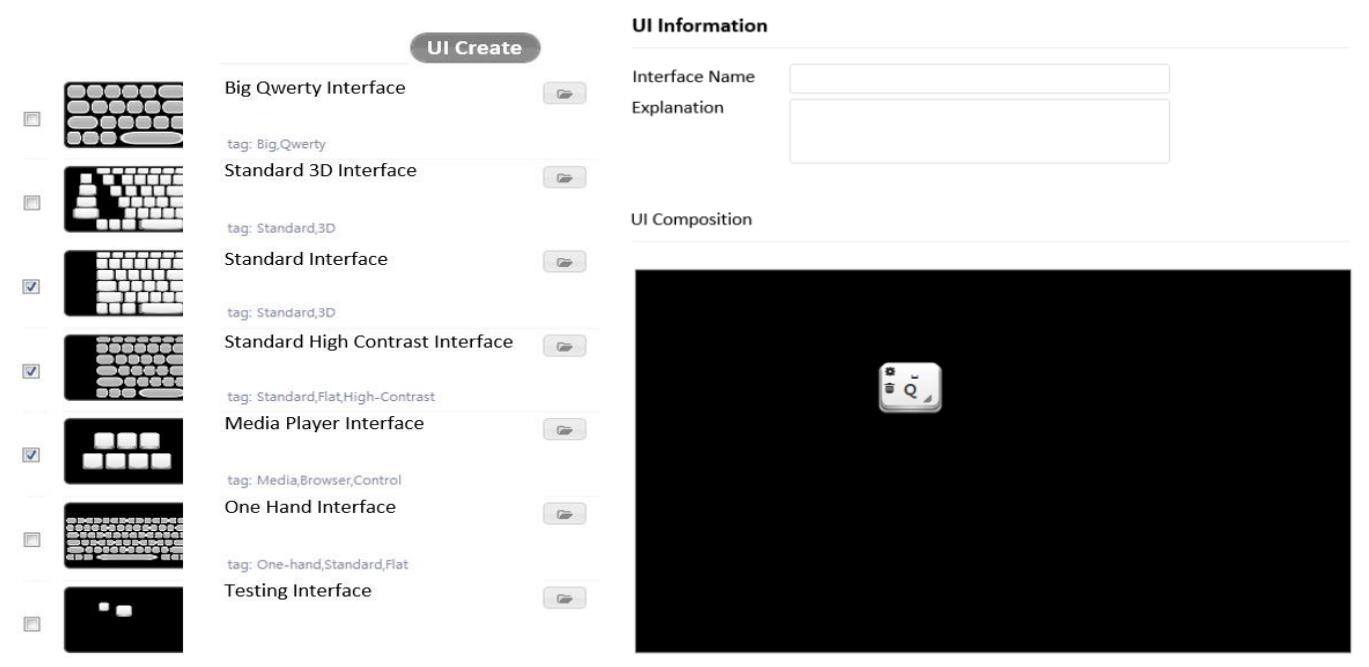

Figure 7. Customized Interface on the Website

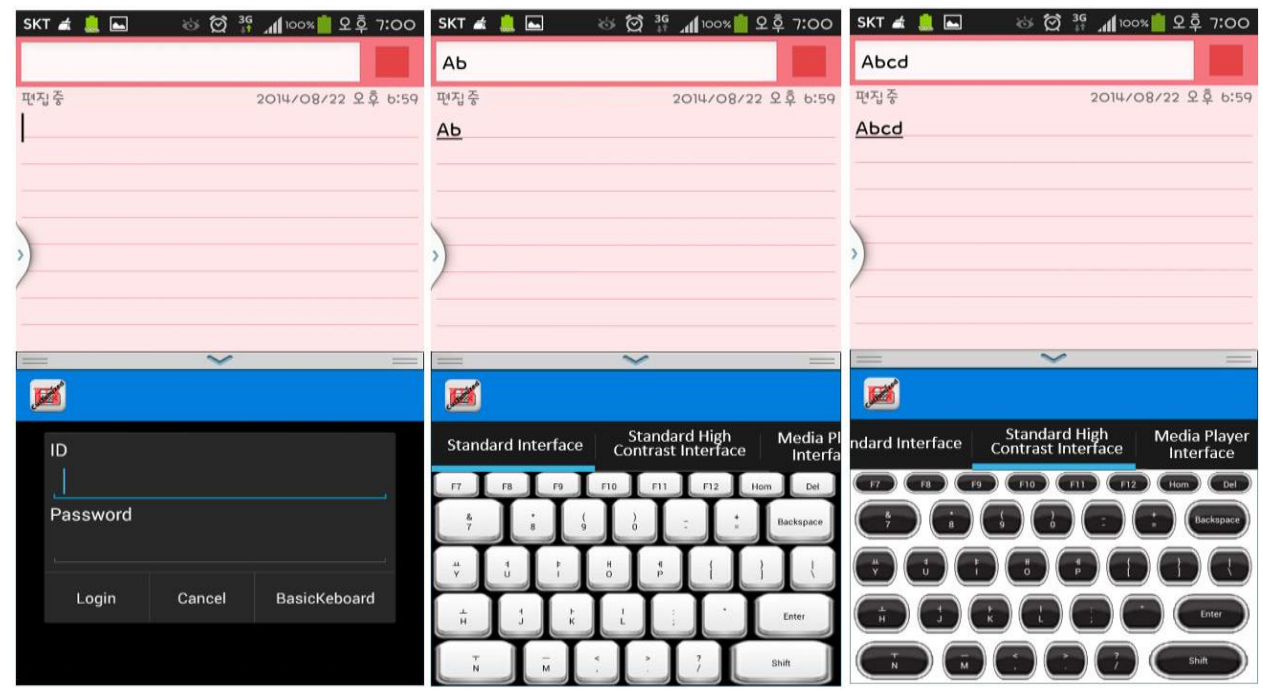

Figure 8. Customized Interface Provided in Smart Mobile

As show in Figure7, the interface customization could be done through web interface and the customized interfaces could be shared among users. Users can use a Web browser interface, provided with the Toolkit from the management server, interface creation. If you enter the user ID and password on the left as shown in the following figure, users or shared with other users, a list of interfaces appears as shown in the illustration. For a list of pictures and easy to see the title of the interface by interface, you can select the desired interface, or you can modify the existing interface and click the Modify button to modify existing production or a new interface can be used. The illustration on the right is the way to create a user interface, the user can save the interface to create a title and description of the interface and the interface directly window appears. The user's interface, using your mouse directly. Once the mouse is pressed, the appearance of a primary key, the user can set the key values, colors, and sizes. Key size and Drag of the mouse interface, Drop function, you can set freely the size, location, easily can be adjusted. When you save, left after the figure produced by the interface's interface is added to the list. Learn from the Web interface, as shown in Figure 8 is 
a smart mobile devices applied to the figure. We have a variety of interface to apply a customized smart mobile devices Samsung Galaxy S4 user interface. By default, the Android keyboard layout, change or modify the code in real time, has been unable to do. Therefore, the dual-way applications can execute select we have developed smart mobile application. The upper-left corner of the figure 8 , as shown in the following figure, after entering username and password, the login button, the server sends the user's interface to request data. Servers are user-managed servers, managed servers, managed servers, interface mapping to retrieve data, and then transmit the desired user interface. You want to apply to the smart mobile device interface for Web based.

\section{Cloud Services for Providing Interfaces}

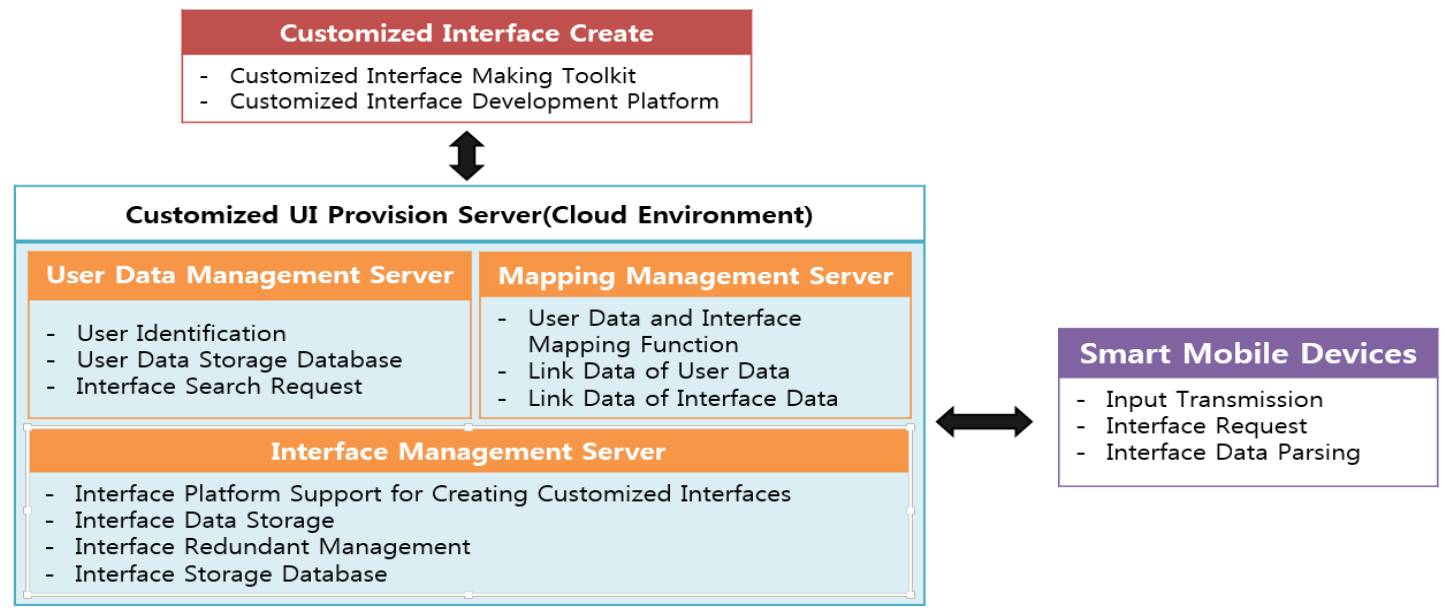

Figure 9. Cloud Structure for Providing Customized Interface

Figure 9 shows a cloud environment providing a user custom interface. Each of the servers will work with each other, as shown in the illustration and each of these features has been included. There are 3 servers in the cloud as shown in the illustration are linked together, the user data management server saves information for identifyingusers, and when you request data through user login, it checks whether you are a registered user, and after retrieves the requested interface data. The mapping management server will request data on the interface. Mapping data from the management server to interface with the user data is mapped to each other link data, and only with a user's ID and interface ID consists of the data because the data can be found as soon as possible. In addition, because of the full interface to manage the mapping data, data that has duplicates. Therefore, link data saves space because they have available more efficiently. Learn the management server, user interfaces, the ability to save interface and the interface is stored as data redundancy is not a waste of storage space and the ability to manage. In addition, allows you to easily create your own interface for a Web-based interface provides an authoring tool, platforms and formats. This uses three servers, user customizable interface provided anytime, anywhere. Smart mobile devices are installed by the user through user-customized interface applications and the pad when you send from the server by typing the Word interface to transfer data. Received data is sent to the user's smart mobile devices, it will try to parse the data so that it can recognize and be then shown to the user. 


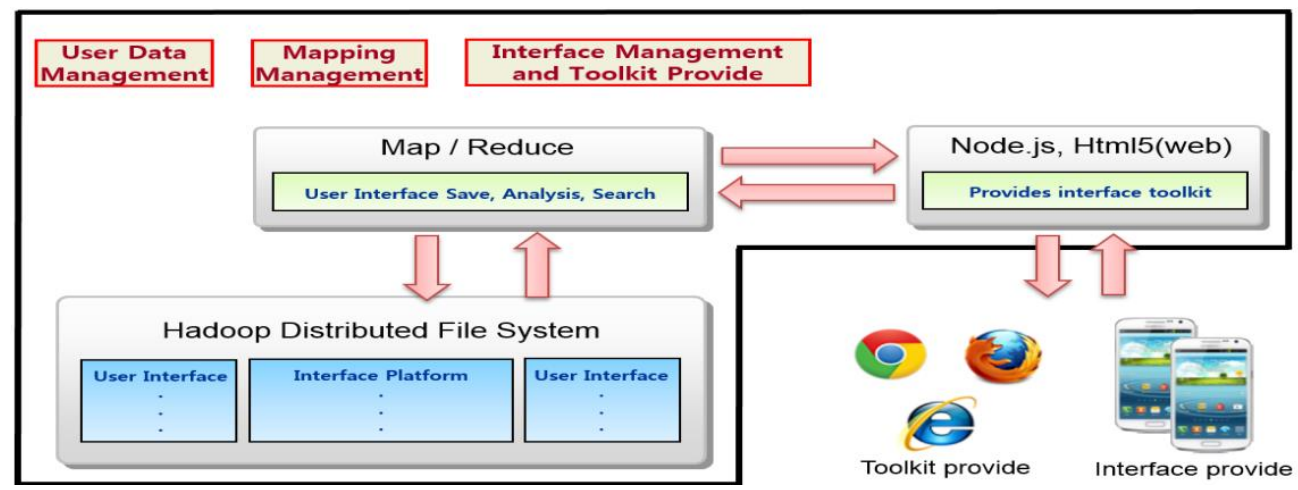

Figure 10. Hadoop Distributed ArchitectureforCustomized Interface

Cloud environments are organized based on Hadoop [7, 8]. Figure 9 servers as shown in Figure 10 consists of the Hadoop distributed file system (HDFS) [9, 10], each server are data stores data in the form of distributed file system. Hadoop distributed file system is copied to the storage and management of data to multiple, because even if the data loss can recover quickly. When you save the data to be stored in HDFS [11, 12], Hadoop's Map when you read the information and reduce the ability to quickly analyze the data and search interfaces that are produced $[13,14]$. User information and interface as the value of the Key and Value data information sharing. Interface, the data is in XML format and is provided to the user, resulting in the user interface, as shown in table 1, data information transmission. When you request data from the user-interface, XML format, but XML File transfer of data in the form of a non-JSON file $[15,16]$. JSON file format is XML File generates less data than capacity, fast data transmission. Smart mobile devices are transferred from the JSON file to the server after you have received is presented to the user through the analysis. Interface Toolkit based on HTML5 [17, 18] and Node.js $[19,20]$ is provided through the Web, there is no need to install a separate program. The user from the server at any time through a Web browser interface can be offered production toolkit.

Table 1. Interface Data From

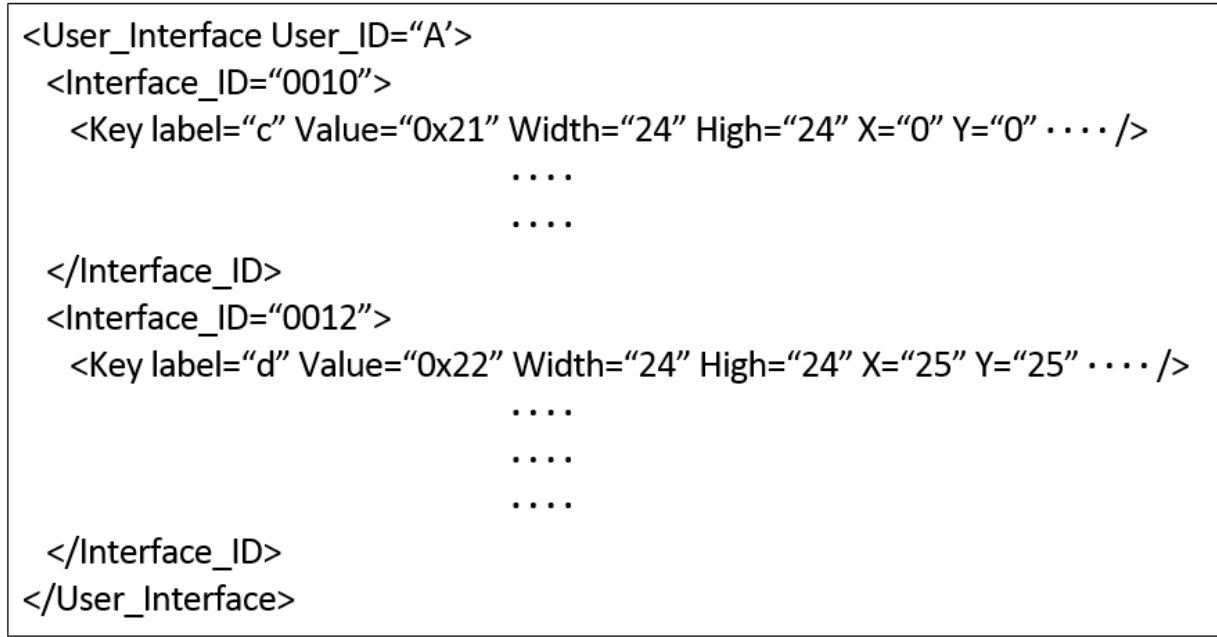

As the mobile device display a variety of smart devices differ. Therefore, in order to apply for a variety of devices, interfaces, as shown in Table 2 and the calculations made using 
scales of the interface. $K_{\text {widt }}$ is a Web authoring tool designed for the size of the keyboard and the display size of mobile devices, the user $D_{\text {widt }}$ says. Usually the vertical direction using a mobile device landscape. $S_{\text {width }}$ is a Web authoring tool is a user-horizontal resolution, $R_{\text {width }}$ is applied to a key-sized mobile devices landscape value. Vertical value will be the same size as the above calculations, even mobile devices have different display sizes calculated in the table expression to apply. For example, Web authoring tools are called the horizontal size of the keyboard and one made in the $60 \mathrm{~s}$, when the Web authoring tools resolution is $1280 \times 720$. The user mobile device used in the resolution of $1920 \times 1080,60 \times 1920 / 1280$ when calculated by the size of the interface is to be applied to 90 .

$$
\begin{aligned}
& K_{\text {width }} \bullet D_{\text {width }} / S_{\text {width }}=R_{\text {width }}(1) \\
& K_{\text {width }} \bullet D_{\text {width }} / S_{\text {width }}=R_{\text {width }}(2)
\end{aligned}
$$

\section{Sharing Interface using Sensor Network}

Figure 11 and 12 demonstrates how the mobile use Gyro sensor and Wi-Fi direct interface to share data. Latest Wi-Fi Direct allows so much to the technology used in smart mobile devices, without the AP connected via a direct connection between the device data sharing, synchronization, transfer easily. Because of the use of techniques based on Wi-Fi faster than Bluetooth, interfaces can transfer data reliably. They is stored in the smart mobile devices provided by the server before the user, as shown in Figure 11 is a custom interface, and transmits the data to the data transfer interface when JSON file format. XML is similar to JSON file format, but the capacity of data can be transmitted rapidly reduced. When data is transferred from the interface JSON file, and stores the data in internal memory interface analyzes it. It may be saved by selecting the interface at any time. Figure 12 explains the process when mobile device uses Wi-Fi Direct on docking station. By default almost all modern smart devices have Gyro sensor which can detect the action of device quickly sharing from left to right repeat.

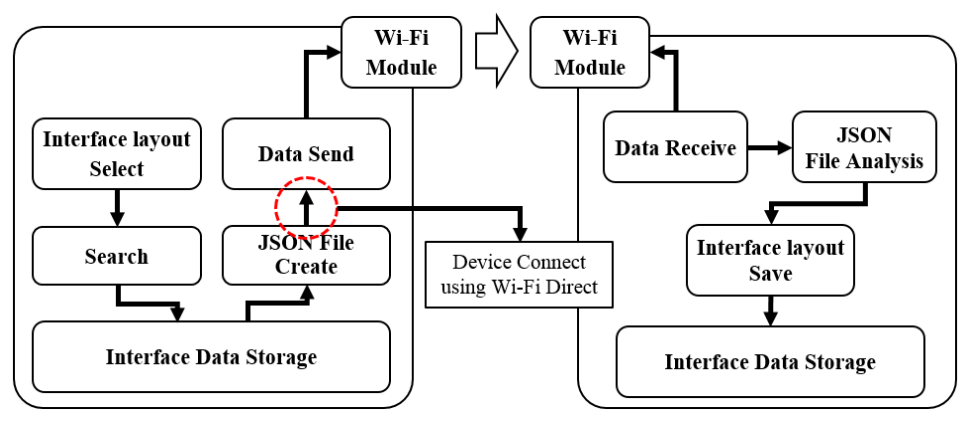

Figure 11. Interface Data Sharing Method 


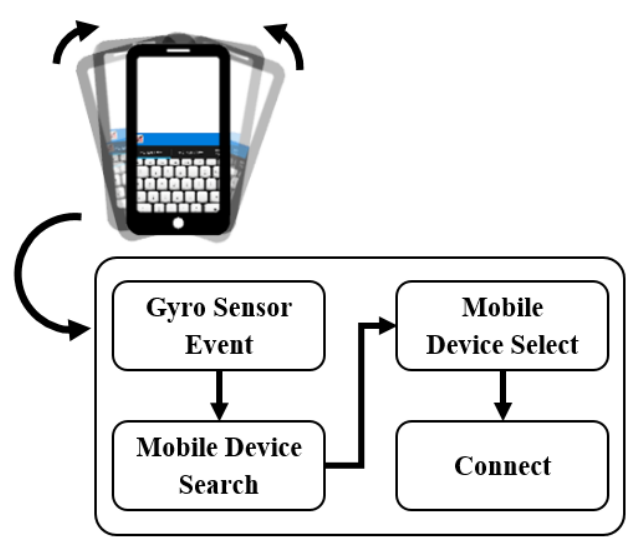

\section{Figure 12. Interface Data Sharing Method}

We use the following expression for checking rapidly changing the angle of the Gyro Sensor value, and look for other user's smart device. Gyro sensor obtain the acceleration sensor of the moment based on the value of the base point value is to calculate a value up or down. We use equation 3 and 4 in order to obtain Gyro sensor value. In equation, $M_{r e s}$ is the high-resolution (resolving power), $V_{r e f}$ is voltage of Gyro Sensor, $S_{\text {sen }}$ is sensitivity ofGyro Sensor, $S_{\text {res }}$ is the values of the speed of the time there has a change in the full resolution is $1, S_{d e g}$ is the rotation angle of the sensor.

$$
\begin{gathered}
\frac{V_{\text {ref }}}{M_{\text {res }}}\left[\frac{m V}{b i t}\right] * \frac{1}{S_{\text {sen }}\left[m V /\left(^{\circ} / S\right)\right]}=S_{\text {res }}\left[\frac{\left(^{\circ} / S\right)}{\text { bit }}\right] \\
S_{\text {res }}\left[\frac{(\% / S)}{\text { bit }}\right] * \frac{\pi}{180^{\circ}}=S_{\text {deg }}\left[\frac{(\mathrm{rad} / \mathrm{s})}{\text { bit }}\right]
\end{gathered}
$$

For example, if a smart mobile device has resolution of 1024, Gyro sensor voltage scale of $3.3 \mathrm{~V}$, sensitivity of $2 \mathrm{mV}$; the $3 \mathrm{rd}$ and 4 th expressions are applied.

$$
\begin{aligned}
& \frac{3300}{1024}\left[\frac{\mathrm{mV}}{b i t}\right] * \frac{1}{2\left[\frac{\mathrm{mV}}{\left.\mathrm{o}^{\circ} \mathrm{S}\right)}\right]}=1.611\left[\frac{\left.\mathrm{(}^{\circ} / \mathrm{S}\right)}{b i t}\right] \\
& 1.611\left[\frac{\left.\mathrm{(}^{\circ} / \mathrm{S}\right)}{\mathrm{bit}}\right] * \frac{\pi}{180^{\circ}}=0.028\left[\frac{(\mathrm{rad} / \mathrm{s})}{\mathrm{bit}}\right]
\end{aligned}
$$

In this way, based on the calculated value "if(Before degree $>$ Current degree $\left.+3^{\circ}\right)$ " or "if(Before degree < Current degree- $\left.3^{\circ}\right)$ ". When the event meets the condition by repeatedly, the other party smart mobile device isfound, and then user can select the device to connect to. From Figure 11 we can see the process of data transmitting by WiFi Direct. On the receiver side, the devices will analyzing the memory to save the data before receive the XML format file. Of course by default, the layout data can be share through the cloud server but it requires Internet connection. And in case the users prefer sharing immediately, using Wi-Fi Direct method is much more convenient and speedy.

\section{Conclusion}

This paper present the use of cloud services in customizing mobile interface. The proposed systems can offer various customized interfaces for the users. To eliminate the action of finding and installing themes again and again in case user has multiple device, by utilizing cloud services the set up part is only a simply login. By providing many 
typed and highly customized interface, useful layout for the elderly, the disabled, children etc. could be put to use in various environment. In the future, hopefully more extensive research on this project could bring out better result.The current application of this paper supports dual display on only supported smart mobile devices. Thus, future research will focus on usage on smart mobile devices that do not support dual display to make it easy to usage.

\section{Acknowledgements}

This research was financially supported by the MSIP (Ministry of Science, ICT \&Future Planning), Korea Evaluation Institute of Industrial Technology Research Program, Korea 2014, the MSIP (Ministry of Science, ICT and Future Planning), Korea, under the ITRC (Information Technology Research Center) support program (NIPA-2014-H0301-14-1014) supervised by the NIPA(National IT Industry Promotion Agency) and Prof. Ilmin Kim was financially supported by Hansung University.

\section{References}

[1] "Emarketer", http://www.emarketer.com.

[2] S. Allen, V. Graupera and L. Lundrigan, "The Smartphone is the New PC", Pro Smartphone Cross-Platform Development. Apress, (2010), pp. 1-14.

[3] E. Y. C. MistutakaItoh, "Virtual smartphone over IP", 2010 IEEE International Symposium on World of Wireless Mobile and Multimedia Networks, (2010) June 1-6, Montrreal, Canada.

[4] M, Romero, C, Southern, B, Frey and G. D. Abowd, "Braille Touch: designing a mobile eyes-free soft keyboard", Proceedings of the 13th International Conference on Human Computer Interaction with Mobile Devices and Services. ACM, (2011) August 707-709, New York, USA.

[5] J. Wu, "A framework for learning comprehensible theories in XML document classification", IEEE Transactions on Knowledge and Data Engineering, vol. 24, no. 1, (2012), pp. 1-14.

[6] T. N. Le, H. Wu, T. W. Ling, L. Li and J. Lu, "From Structure-Based to Semantics-Based: Towards Effective XML Keyword Search", Conceptual Modeling. Springer Berlin Heidelberg, vol. 8217, (2013), pp. 356-371.

[7] J. Dittrich and J.-A. Quiane-Ruiz, "Efficient big data processing in Hadoop Map Reduce", Proceedings of the VLDB Endowment, vol. 5, no. 12, (2012), pp. 2014-2015.

[8] H. Tan, W. Luo and L. M. Ni, "CloST: a Hadoop-based storage system for big spatio-temporal data analytics", Proceedings of the 21st ACM international conference on Information and knowledge management. ACM, (2012), October 2139-2143, New York, USA.

[9] D. Guo, Y. Du and L. Hu, "HDFS Based Cloud Data Backup System" Journal of Jilin University (Science Edition), vol. 50, no. 1, (2012), pp. 101-105.

[10] S. Daneshyar and M. Razmjoo, "Large-Scale Data Processing Using Map reduce in cloud Computing Environment", International Journal on Web Service Computing (IJWSC), vol. 3, no. 4, (2012), pp. 1-13.

[11] D. Guo, W. Shi and M. Hou, "Improvement and Implementation of Hadoop HDFS Model in Private Cloud", Proceedings of the 9th International Symposium on Linear Drives for Industry Applications, Springer Berlin Heidelberg, vol. 4, (2014), pp. 663-670.

[12] H. J. Singh and V. P. Singh, "High Scalability of HDFS using Distributed Name space", International Journal of Computer Applications, vol. 52, no. 17, (2012), pp. 30-37.

[13] J. Dean and S. Ghemawat, "Map Reduce: simplified data processing on large clusters", Communications of the ACM, vol. 51 no. 1, (2008), pp. 107-113.

[14] R. Vernica, M. J. Carey and C. Li, "Efficient parallel set-similarity joins using Map Reduce", Proceedings of the 2010 ACM SIGMOD International Conference on Management of data. ACM, (2010), June pp. 495-506, New York, USA.

[15] K. Maeda, "Performance evaluation of object serialization libraries in XML, JSON and binary formats", Digital Information and Communication Technology and it's Applications (DICTAP), 2012 Second International Conference on IEEE, (2012), May, pp. 177-182, Bangkok, Thailand.

[16] A. Sarasa-Cabezuelo and J.-L. Sierra, "Grammar-driven development of JSON processing applications", Computer Science and Information Systems (FedCSIS), 2013 Federated Conference on. IEEE, (2013), September, pp. 1557-1564, Krako, Poland.

[17] D. Akhawe, F. Li, W. He, P. Saxena and D. Song, "Data-confined html5 applications", Computer SecurityESORICS 2013. Springer Berlin Heidelberg, vol. 8134, (2013), pp. 736-754. 
[18] R. Gawley, J. Barr and M. Barr, "Native to HTML5: A Real-World Mobile Application Case Study", Mobile Computing, Applications, and Services. Springer Berlin Heidelberg, vol. 95, (2012), pp. 188-206.

[19] J. Duda and W. Dłubacz, "Distributed evolutionary computing system based on web browsers with javascript", Applied Parallel and Scientific Computing. Springer Berlin Heidelberg, vol. 7782, (2013), pp. 183-191.

[20] A. G. Hernandez and M. N. M. Garcia, "Restful Triple Space Management of Cloud Architectures", 7th International Conference on Knowledge Management in Organizations: Service and Cloud Computing. Springer Berlin Heidelberg, vol. 172, (2013), pp. 571-579.

\section{Authors}

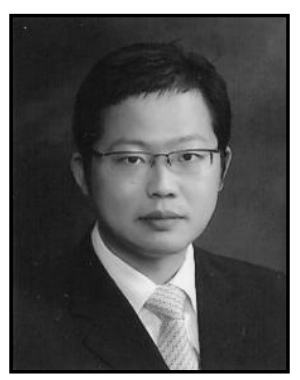

Sanghyun Park received his B.S. Degree in Computer and Information from the University of Korea Nazarene. He worked as an engineer in System Development Team of Media Flow Company from 2010 to 2012. He is now studying Master Degree in Electronics and Computer Engineering at Chonnam National University. His research interests are Interactive Media, Systems Development, Embedded of systems, Digital Media and Cloud computing.

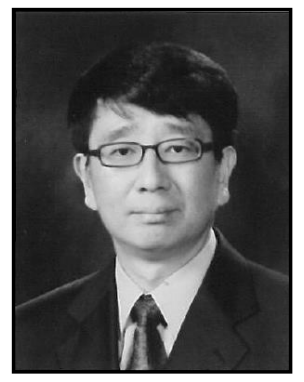

IlminKim received a B.S. degree in Computer Engineering from KyungBuk National University in 1984 and a Ph. D degree from Arizona State University. He is currently a Professor at Department of Computer Engineering, Hansung University, South Korea. His current Research interests include distributed computing and operating systems.

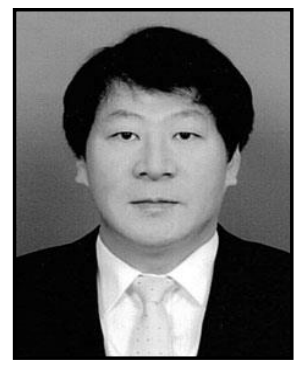

Jinsul Kim received the B.S. Degree in computer science fromUniversity of Utah, Salt Lake City, Utah, USA, in 2001, and theM.S. and Ph.D degrees in digital media engineering, departmentof information and communications from Korea Advanced Instituteof Science and Technology (KAIST), Daejeon, South Korea, in2005 and 2008. He worked as a researcher in IPTV InfrastructureTechnology Research Laboratory, Broadcasting/TelecommunicationsConvergence Research Division, Electronics and TelecommunicationsResearch Institute (ETRI), Daejeon, Korea from 2005 to 2008. Heworked as a professor in Korea Nazarene University, Chon-an, Koreafrom 2009 to 2011. Currently, he is a professor in Chonnam NationalUniversity, Gwangju, Korea. He has been invited reviewer for IEEE Trans. Multimedia since 2008. His research interests include QoS/QoE, Measurement/Management, IPTV, Mobile IPTV, Smart TV, Multimedia Communicationand Digital Media Arts. 